\title{
Author Correction: The discovery of Bombali virus adds further support for bats as hosts of ebolaviruses
}

Tracey Goldstein (D), Simon J. Anthony, Aiah Gbakima, Brian H. Bird, James Bangura, Alexandre Tremeau-Bravard, Manjunatha N. BelaganahalliD, Heather L. Wells D, Jasjeet K. Dhanota D, Eliza Liang, Michael Grodus, Rohit K. Jangra, Veronica A. DeJesus, Gorka Lasso, Brett R. Smith, Amara Jambai, Brima O. Kamara, Sorie Kamara, William Bangura, Corina Monagin, Sagi Shapira, Christine K. Johnson, Karen Saylors, Edward M. Rubin, Kartik Chandran, W. Ian Lipkin and Jonna A. K. Mazet

Correction to: Nature Microbiology https://doi.org/10.1038/s41564-018-0227-2, published online 27 August 2018.

In the version of this Article originally published, the bat species for 12 individuals were incorrectly identified in Supplementary Table 1 and 2. After resequencing the $M T-C y t B$ and $M T$-CO1 segments and reviewing the data, the authors have corrected the errors for these 12 animals. In the amended version of the Supplementary Information, Supplementary Tables 1 and 2 have been replaced to include the corrected host species information. None of the 12 bats affected were positive for the Bombali virus, and the conclusions of the study are therefore unchanged.

Published online: 8 November 2018

https://doi.org/10.1038/s41564-018-0315-3 